A N N A L E S Annales de Bretagne et des Pays de l'Ouest

Anjou. Maine. Poitou-Charente. Touraine

123-4 | 2016

Varia

\title{
Inventer un regard
}

\section{David Bensoussan}

\section{OpenEdition}

Journals

Édition électronique

URL : http://journals.openedition.org/abpo/3463

DOI : $10.4000 /$ abpo.3463

ISBN : 978-2-7535-5365-1

ISSN : 2108-6443

Éditeur

Presses universitaires de Rennes

Édition imprimée

Date de publication : 30 décembre 2016

Pagination : 202-203

ISBN : 978-2-7535-5363-7

ISSN : 0399-0826

Référence électronique

David Bensoussan, «Inventer un regard », Annales de Bretagne et des Pays de l'Ouest [En ligne], 123-4 I

2016, mis en ligne le 30 décembre 2016, consulté le 23 septembre 2020. URL : http://

journals.openedition.org/abpo/3463; DOI : https://doi.org/10.4000/abpo.3463 
Boulouch, Nathalie, ANDRÉ Louis, Inventer un regard. Rennes et la Bretagne à travers les collections de la société photographique de Rennes (1890-1976), Rennes, PUR, $2015,128 \mathrm{p}$.

Créée le 13 juin 1890, à l'hôtel de ville, la société photographique de Rennes (SPR) a commémoré l'an dernier son $125^{\mathrm{e}}$ anniversaire. Commémoration qui s'est, entre autres, traduite par la publication de cet ouvrage destiné à relater brièvement son histoire et à exposer un certain nombre de photographies réalisées par ses membres tout au long de son existence. Des deux introductions, parfois un peu redondantes, on retiendra surtout le parcours historique de cette société photographique dont la fondation relève autant de l'entrée de la photographie dans l'ère industrielle que du vaste mouvement de multiplication des sociétés d'amateurs à la fin du XIX siècle. De fait, deux ans après sa création, la SPR adhère à l'Union nationale des Sociétés photographiques de France, nouvellement créée, participant ainsi à l'animation d'un réseau de plus en plus dense à l'échelle nationale et internationale. Elle devient également membre de l'Union internationale de photographie. Devenue plus accessible depuis la mise au point des appareils portatifs et l'utilisation de plaques prêtes à l'emploi, puis par l'introduction de la pellicule souple, la photographie attire, à Rennes comme ailleurs, un nombre croissant d'amateurs qui, tout en s'intéressant à ses multiples applications, entendent aussi perfectionner leurs pratiques. Comme le souligne Nathalie Boulouch, ces amateurs " éclairés " élaborent, au sein d'une expérience associative spécifique, la construction d'un regard photographique forgé par des références collectives et la volonté de faire accéder la photographie au rang d'un art à part entière. D'origine bourgeoise pour l'essentiel, ils trouvent dans les excursions photographiques que la société organise aux beaux jours, notamment au Boël (en Bruz), sur les bords de la Vilaine, un type de loisir qui leur convient parfaitement. Convivialité et formation par l'échange s'affirment ici dans un cadre propice à l'expérimentation de la maîtrise de la lumière et du calcul du temps de pose.

Progressivement cependant, l'essor de la pratique photographique dans l'entredeux-guerres entraîne une plus grande diversité sociale des membres de la société tandis que l'avènement à la présidence de l'architecte Georges Nitsch en assure le rayonnement. En son sein, les échanges se multiplient entre " anciens " et " nouveaux " tandis que des formations en ateliers ainsi que des cours répondent à une demande croissante d'apprentissage et de perfectionnement. Concours et expositions se développent également, notamment dans les galeries de l'École des beaux-arts de Rennes. On note alors le développement de la couleur avec l'utilisation des autochromes qui séduisent nombre de membres de la SPR et qui font parfois l'objet de projections. Parallèlement, la société s'ouvre au cinéma amateur, s'appuyant là encore sur les progrès techniques en ce domaine. L'intérêt est tel qu'en 1935, elle décide de créer une section cinéma qui adhère à l'Union des amateurs cinéastes de France fondée en 1933. Cette dynamique s'exprime plus nettement encore durant les Trente glorieuses particulièrement avec l'essor de la diapositive qui favorise, surtout dans les années 1960 et 1970, les échanges entre membres autour de " diaporama " associant le son et l'image. Si la multiplication des " photo-club " témoigne de l'éclatement de la scène associative en ce domaine, l'organisation en 1976 des premières " Rencontres photographiques de Rennes ", à l'initiative de son président Pierre Chemin, conforte cependant la place de la SPR dans la cité. C'est d'ailleurs sur cette date que s'arrête ce panorama historique qui, bizarrement, fait l'impasse sur toute la période suivante.

Tout le reste de l'ouvrage est consacré à la présentation de nombreuses photographies émanant des membres de la société depuis sa création. Il faut regretter ici l'absence de classement et la faiblesse des commentaires qui, trop rarement du reste, les accompagnent. Sur ce point, le propos préalable de Nathalie Boulouch ne suffit pas, 
même s'il ouvre des perspectives de classement qui auraient gagné alors à être mises en œuvre et confrontées aux photographies exposées. Nombre de ces réalisations s'inscrivent, en effet, implicitement ou explicitement, dans les préoccupations de l'Union nationale des Sociétés photographiques de France qui entend constituer, par le biais des sociétés qui la composent, les archives pittoresques des provinces. "Documenter le monde proche " est ainsi, sans conteste, le fil directeur d'une bonne partie des photographies exposées. Conservatoire des traditions, la Bretagne se donne donc ici à voir dans de nombreux clichés, dans tous les sens du terme. Bretonnes en coiffe, scènes de la vie religieuse, activités agricoles et maritimes constituent tout un ensemble de réalisations d'inégal intérêt. On remarque l'influence persistante, du moins jusqu'à la veille du second conflit mondial, du courant pictorialiste qui cherche le dialogue avec la peinture. Les effets de lumière sur les bords de mer, les fumées produites par le brûlage du goémon offrent alors des effets visuels qui s'inscrivent dans ce cadre. Certaines photographies de Georges Nitsch, la plupart du temps réalisées sur des plaques de verre au gélatino-bromure d'argent, se singularisent alors par la qualité du coup d'œil et de la maîtrise technique à l'instar de cette scène de labour (p. 33), prise vers 1930, d'où se dégage une image saisissante du rapport de l'homme à la nature.

Ce souci d'inventaire documentaire se perçoit également dans les nombreuses photographies de la ville de Rennes. Au sein de cet ensemble, là encore très inégal, nombre de réalisations n'offrent qu'un intérêt historique, nullement négligeable du reste. Ainsi en est-il des photographies autour du procès Dreyfus ou du champ de course des Gayeulles, à la charnière des $\mathrm{XIX}^{\mathrm{e}}$ et $\mathrm{xx}^{\mathrm{e}}$ siècles. Les clichés sur les funérailles du cardinal Charost, en novembre 1930, puis ceux sur la construction du quartier Colombier ou du square des Hautes Ourmes dans les années 1970 s'inscrivent également dans cette dimension. On leur préférera les clichés dont l'intérêt documentaire se situe dans la capacité à rendre compte de l'atmosphère d'un moment du quotidien, grâce à une qualité artistique qui ouvre des horizons d'observation et de sensation à l'exemple de la vue stéréoscopique d'Ambroise Poirier prise sur les quais (p. 44), le 17 février 1924, ou de cette scène de noce captée par Georges Dorer à la fin des années 1950 depuis les étages de l'Hôtel de ville (p. 52).

Si le regard de l'amateur ne suscite pas toujours l'adhésion, il n'en reste pas moins que cet ouvrage, dont on peut regretter cependant qu'il n'ait pas pris une forme plus aboutie, permet avec intérêt de replacer la pratique photographique dans le temps et dans l'espace d'une ville de province en soulignant les sociabilités spécifiques qu'elle met en œuvre.

David BENSOUSSAN

Molgno, Yves, Histoire du Petit Séminaire de Quintin (1934-1975), Quintin, Association des Anciens élèves du Petit Séminaire de Quintin, 2012, 353 p.

Quarante ans après l'étude pionnière de Jean-René Chotard sur Guérande (1977), le temps semble opportun d'un retour sur l'histoire des Petits Séminaires bretons qui ont joué un rôle éminent dans la formation des jeunes garçons. Que l'éditeur de cet ouvrage sur le Petit Séminaire de Quintin, soit l'Association des anciens élèves, dont l'auteur est lui-même membre actif, ne saurait être anodin : l'entreprise est largement le fruit d'une démarche participative qui a permis de rassembler clichés, témoignages personnels (85), bulletins imprimés... et de compenser heureusement la faiblesse des archives provenant de l'institution. L'ouvrage témoigne donc d'abord d'une démarche de mémoire mais il offre aussi l'occasion d'une véritable enquête 